\title{
Parameter Analysis of Pulsed Eddy Current Sensor using Principal Component Analysis
}

\author{
Faris Nafiah, Mohammad O. Tokhi, Senior Member, IEEE, Gholamhossein Shirkoohi, Member, IEEE, \\ Fang Duan, Zhanfang Zhao, Giorgos Asfis, and John Rudlin
}

\begin{abstract}
Pulsed eddy curent (PEC) technique provides a means to inspect structures without surface contact. It is particularly useful when the structure's surface is rough or inaccessible, such as insulated pipes in pipeline. Probe parameters of a PEC system, especially the sensing and excitation coil diameters, can significantly affect the PEC system's performance. Thus, detailed analysis of these parameters is paramount in developing a PEC system. Currently, this is accomplished by establishing the trend of features with respect to the analyzed variables, e.g. sample thicknesses. However, prior to extracting these features, a number of configuration parameters have to be determined. For this reason, analyzing PEC performance over a range of coil diameter values is rather time-consuming as both the sensing and excitation coil diameters significantly affect the received signals. Principal component analysis (PCA) is proposed as an alternative to the feature extraction. The work here analyzes the trends contributed by the PCA scores for different values of sensing and excitation coil parameters. Results from both numerical simulations and experiments suggest that the sensitivity of the PEC probe is highly correlated with the excitation coil diameter, while the excitation-sensing coil distance is not significant in determining the sensitivity of the PEC probe. These findings are consistent with those reported in the literature, suggesting the potential of adopting PCA for an automated PEC performance analysis process.
\end{abstract}

Index Terms_ parameter analysis, pipeline inspection, principal component analysis, pulsed eddy current

\section{INTRODUCTION}

G IVEN that is a non-invasive and non-contacting nondestructive testing (NDT) technique, pulsed eddy current (PEC) has been widely used in pipeline inspection and aircraft industries. Similar to other NDT methods, the performance of PEC depends on many factors. The sensitivity of a PEC probe, for example, is proportional to its diameter [1]. Meanwhile, the same configuration parameter is inversely proportional to the range of measurement, or the depth of penetration [2]. Varying the coil height and width, on the other hand, has been proven to offer better sensitivity [3]. These influences of parameters are only applied under the condition where the excitation current is kept unchanged. Varying the excitation current parameters, through its excitation frequency and edge time of the pulse wave, have negative effects on the depth of penetration [4]. Because of such high dependency of PEC performance on various variables, parameter analysis plays a critical role in the design process of a PEC system.

The work here aims to facilitate the automation of the design process of PEC probe, specifically for pipeline inspection, by virtue of simplifying the feature extraction process.

This work was supported by Lloyd's Register Foundation.

F. Nafiah is with the School of Engineering, London South Bank University and TWI Ltd. (e-mail: nafiahf@Isbu.ac.uk).

Mohammad O. Tokhi, Gholamhossein Shirkoohi, Duan Fang and Zhanfang Zhao are with the School of Engineering, London South Bank University (e-mail: tokhim@Isbu.ac.uk).

Giorgos Asfis and John Rudlin are with TWI Ltd. (e-mail: giorgos.asfis@twi.co.uk).
The scope here is limited to ferromagnetic material application, specifically carbon steel, since feature interpretation for ferromagnetic material is different from non-ferromagnetic material. However, it is believed that the approach proposed here can still be applied to non-ferromagnetic materials. PEC architecture under study is the pancake-type transmit-receive coil configuration, since this configuration has been common in quantifying pipe wall thickness [5]. The design process of a PEC probe for pipeline inspection needs to consider the influences of probe parameters with respect to the test specimen thickness. Conventionally, the process of assessing the performance of a PEC system is generally accomplished by evaluating the trend of features with respect to wall thicknesses [6], [7]. However, particularly in case of pipeline inspection, the conventional decay transition time, $\tau_{0}$, which is used for thickness quantification, possesses several challenges. The extraction of $\tau_{0}$ from PEC measurement signals requires determining multiple parameters in order to establish the reference line [8], [9]. This requires strict analysis of the shape of the reference signal. This process is heuristic, making the assessment to be rather qualitative. At the same time, the reference line has to be re-established whenever the analyzed parameters are changed, preventing the performance analysis process of a PEC system to be automated. Although a feature extraction based on the signal gradient [5], [10] has recently been proposed, it still requires establishing the amplitudegating parameters by monitoring the segment where the PEC signal behaves linear-like in a semi-logarithmic plot. This 
poses a problem when dealing with different probe parameters, e.g. outer diameter, as the amplitude of the received PEC signal can change in accordance with the probe parameter. In return, amplitude-gating parameters have to be carefully determined for each set of PEC signal received from each analyzed parameter, thus complicating the performance analysis of a PEC probe.

Although the use of optimization algorithm for eddy current testing (ECT) has been reported in the literature, to the authors' knowledge, only Faurschou et al. [11] have proposed a performance analysis tool for PEC. They have proposed a hybrid use of projection to latent structure (PLS) and variable importance in the projection (VIP) to provide a quantitative analysis of the influences of probe parameters on the received PEC signals [11].

This paper opens up the use of principal component analysis (PCA) as a means of feature extraction to eliminate the need to declare preliminary configuration parameters. This, in return, enables the performance analysis of PEC to be automated to ease the PEC probe design process. Although the use of PCA is not new in the area of PEC [12]-[15], it has not been used for pipeline inspection, nor for performance analysis. PCA is capable of assessing the variations between the dimensions of a given data set. Thus, the paper presents an attempt to exploit this PCA characteristic for variation quantification of different data sets, given different values of probe parameters, e.g. sensing and excitation coil outer diameter.

\section{BACKGROUND KNOWLEDGE}

\section{A. PEC Principle}

PEC operates by exciting rectangular-shaped current into a coil placed over a test piece. This supplies magnetic field to the test piece, which consequently results in an induction of eddy current in the test piece, occurring during the falling/rising edge of the coil current. The eddy current is then diffused over time due to the test piece's resistance. This effect can be observed from the changes in the PEC sensing responses. For ferromagnetic material, the diffusion effect is more pronounced when using a sensing coil instead of a magnetic sensor due to the low dynamic range of most magnetic sensors [16]. Typical PEC signal for a ferromagnetic material application is plotted in semi-logarithmic plot, as shown in Fig. 1(b) (transformed using bi-symmetric logarithmic transformation).

\section{B. Problem Formulation}

In the design stage of a PEC probe for pipeline inspection, performance analysis is typically carried out for a specific range of analyzed probe parameters. The parameter focused upon here is the coil diameter, since it shows the most significant influence of the probe performance, in terms of sensitivity and depth of penetration [1], [11]. Both excitation and sensing coil diameters are considered. Since the excitation coil is positioned within the sensing coil, changing the diameter of one coil should change the diameter of another. To avoid the co-dependency of the two parameters, when changing the excitation coil diameter, all parameters of the sensing coil as well as the excitation coil width are fixed. The excitation
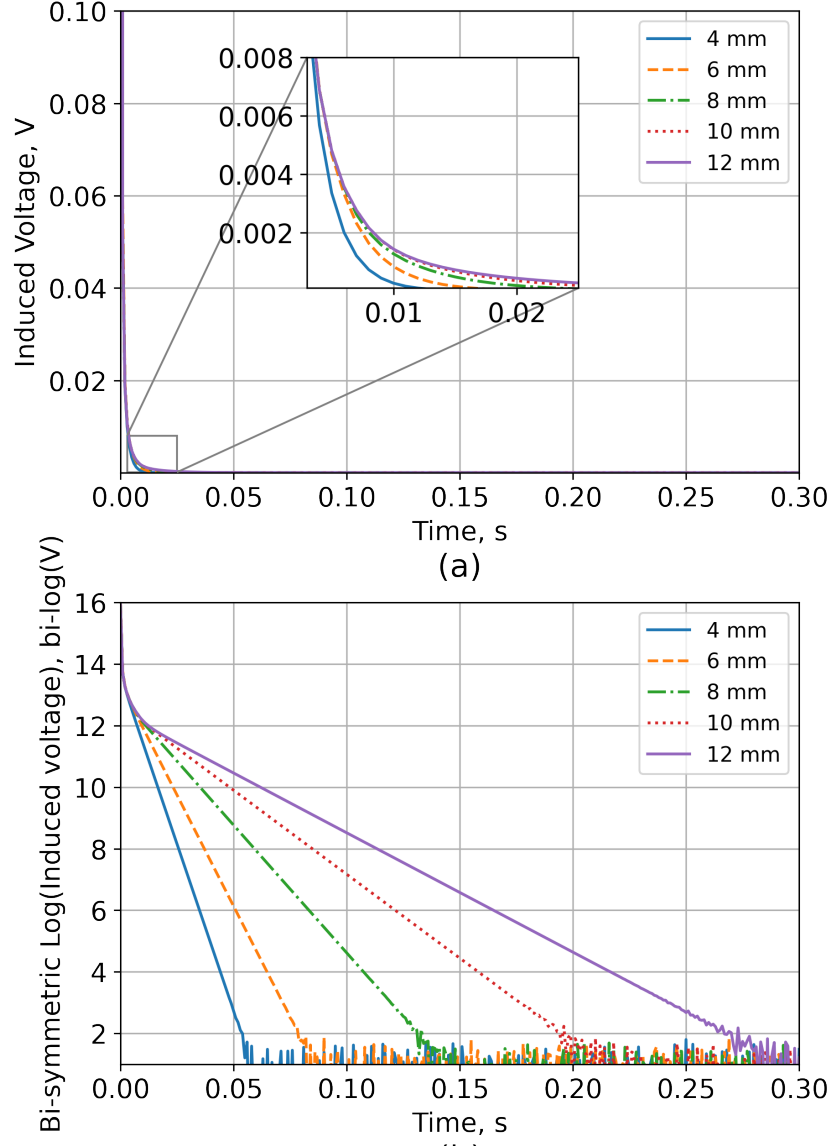

(b)

Fig. 1. Plot of raw PEC signals in a (a) linear plot, and (c) bi-symmetric transformed PEC signals (equivalent to semi-logarithmic plot).

coil diameter is re-phrased as 'excitation-sensing coil distance' $\left(\delta_{\text {exc-sen }}\right)$. On the other hand, when changing the diameter of the sensing coil, $\delta_{e x c-s e n}$ and $w_{s}$ are fixed, so that the outer diameters of both excitation and sensing coils are varied. For ease of reading, sensing coil outer diameter is denoted as $O D$. Figure 2 shows a visualisation of the probe for clearer explanation of these two parameters. As seen in the figure, both coils share the same parameters: inner diameter, $d i$, outer diameter, $d o$, width $w$, and height, $h$. The subscripts notations, $e$ and $s$, represent the excitation coil and sensing coil, respectively. The unit used here is millimeter $(\mathrm{mm})$. The width, $w$, can be represented by the equation $w=d o-d i$. The value of $\delta_{\text {exc-sen }}$ can be defined as $\delta_{e x c-s e n}=d i_{e}-d o_{s}$. When varying the value of $\delta_{\text {exc-sen }}$, the parameters $d o_{e}, d i_{e}, w_{e}$, and $w_{s}$ are kept constant at their respective reference values, while $d o_{s}$ is varied using the equation $d o_{s,(r e f)}-\delta_{e x c-s e n}$ (hereinafter, subscript $r e f$ represents the reference value of the parameter). Since $w_{s}$ is kept constant, $d i_{s}$ is also varied by using the equation $d i_{s,(r e f)}-\delta_{e x c-s e n}$. On the other hand, in varying the value of $O D, w_{e}, w_{s}$, and $\delta_{e x c-s e n}$ are kept constant at their respective reference values. Increasing the value of $O D$ by a certain amount, for example $x \mathrm{~mm}$, will make $d o_{e}, d i_{e}, d o_{s}$, and $d i_{s}$ parameters to change in accordance to these relations, respectively: $d o_{e,(r e f)}+x, d i_{e,(r e f)}+x, d o_{s,(r e f)}+x$, and 
$d i_{s,(r e f)}+x$. Throughout the analysis, the excitation current parameters are fixed to only allow the $O D$ and $\delta_{e x c-s e n}$ to influence the performance.

For each analyzed value of $O D$ and $\delta_{\text {exc-sen }}$, a set of PEC signals are obtained, where each PEC signal corresponds to different wall thickness. Performance analysis is made by evaluating the degree of dissimilarity between the signals in each signal set. In this study, a set of PEC signals obtained by varying the pipe wall thickness with a probe parameter $q$ containing $N$ number of $p$-samples of signals (represented by the row vector $\mathbf{v}_{(n)}(q)$ ) is assigned as $\mathbf{V}(q)$. From this, the assumed quantified value of the degree of dissimilarity corresponding to each $\mathbf{v}_{(n)}(q)$ as compared with the other signals in the data set is given as $\theta\left[\mathbf{v}_{(n)}(q)\right]$, while the collective degree of dissimilarity for the PEC signals set $\mathbf{V}(q)$ can be formulated as $\Theta[\mathbf{V}(q)]$. Note that the functional form of $\theta\left[\mathbf{v}_{(n)}(q)\right]$ does not necessarily need to be the same as the formulation of $\Theta[\mathbf{V}(q)]$, but can be related by $\Theta[\mathbf{V}(q)]=$ $f\left\{\theta\left[\mathbf{v}_{(n)}(q)\right]\right\}$, where $f(*)$ is an arbitrary function. Various choices of quantifying the degree of dissimilarity for both $\theta\left[\mathbf{v}_{(n)}(q)\right]$ and $f(\cdot)$ lead to different quantification capability and analysis, but the value of $q$ that gives the maximum probe performance has to satisfy

$$
\hat{q}=\underset{q}{\arg \max } \Theta[\mathbf{V}(q)] .
$$

In the conventional method of using feature extraction [1], [6], feature is extracted for each $\mathbf{v}_{(n)}(q)$ to provide the value of $\theta\left[\mathbf{v}_{(n)}(q)\right]$, while the measure of relative variation of the features corresponding to different sample thicknesses provides the value of $\Theta[\mathbf{V}(q)]$. From (1), it is also evident that in deriving the PEC probe performance, the PEC signals obtained using the chosen value of either $O D$ and $\delta_{e x c-s e n}$ have to be processed to derive the corresponding feature values, such as the decay transition time, $\tau_{0}$ and the signal gradient for ferromagnetic application. As these features require multiple parameters to be determined, where each parameter also plays a vital role in contributing to the PEC performance, PCA is proposed here to eliminate the need to deliberately establish the optimal configuration parameters in the feature extraction stage.

\section{Feature Extraction Using PCA}

The proposed feature extraction method applies PCA to each data set. For each change in the value of the probe parameter, one data set $\mathbf{V}(q)$ is obtained. The projected data samples of each row vector $\mathbf{v}_{(n)}$ of $\mathbf{V}(q)$ onto the new subspace (commonly known as PCA scores) are given as $\mathbf{u}_{(n)}=\left(u_{1}, \ldots, u_{l}\right)_{(n)}$, where $l<p$ so that the dimension of the reproduced data point is significantly reduced. A $p$ dimensional weight vector of the $k$-th component, $\mathbf{w}_{(k)}=$ $\left(w_{1}, \ldots, w_{p}\right)_{(k)}$ is assigned to project each row vector $\mathbf{v}_{(n)}$ onto the new subspace using

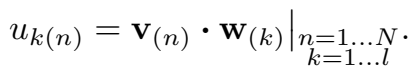

Only the direction of the vector is important for $\mathbf{w}$, not its length. Hence, $\|\mathrm{w}\|=1$. In most PCA implementations, the signal average is subtracted out of the matrix before covariance matrix calculation, as was first applied by [17], [18]. The obtained PCA scores with this approach give the relative distinction of each signal to the projected subspace. This subsequently provides a relative performance assessment of the probe parameters without the need to transform the obtained data, such as normalisation or percentage difference, as was done in the conventional parameter analysis such as in [1].

The value of the first weight $\mathbf{w}_{(1)}$ is obtained by maximising the variance of the projected data samples. Mathematically, this can be expressed as

$$
\mathbf{w}_{(1)}=\underset{\|\mathbf{w}\|=1}{\arg \max }\left\{\sum_{n}\left(\mathbf{v}_{(n)} \cdot \mathbf{w}\right)^{2}\right\} .
$$

Considering the unit vector property of $\mathbf{w}_{(1)}$, (3) can be rewritten in matrix form as

$$
\mathbf{w}_{(1)}=\arg \max \left\{\frac{\mathbf{w}^{T} \mathbf{V}(q)^{\top} \mathbf{V}(q) \mathbf{w}}{\mathbf{w}^{T} \mathbf{w}}\right\} .
$$

From (4), the term in the curly bracket can be maximised by finding the largest eigenvalue of the matrix $\mathbf{V}(q)^{\top} \mathbf{V}(q)$, which incidentally makes $\mathbf{w}$ to be the corresponding eigenvector. Interested reader can derive the expressions for further components, but as the work here aims at finding the separation measure between each response, only the first component is considered. In making sense of PCA scores, $\mathbf{u}_{(n)}$ can also be interpreted as the value the new projected data samples possess in the new principal component coordinate system.

PCA is proposed as a means to transform the original signal into a number of uncorrelated variables. These variables account for as much of the variability in all of the PEC signals as possible, which can be useful to measure how distinct PEC signals are from the reference signal. A comprehensive explanation of PCA can be obtained elsewhere (see for example [19]), while the explanation here is focusing on how maximising the projections of the original data samples onto the new subspace can be utilised to find the degree of separation between received signals and the reference signal. From (2), given the formulation of $\mathbf{w}_{(1)}$ in (4), the respective signals dissimilarity measures of $\theta\left[\mathbf{v}_{(n)}(q)\right]$ in Section II$\mathrm{B}$ can then be formulated as $\mathbf{u}_{1}$. As mentioned before, the interpretation provided by $\mathbf{u}_{1}$ should therefore provide the optimal probe parameter values, which should eventually serve the purpose of maximising $\Theta[\mathbf{V}(q)]$ shown in (1).

Data pre-processing is essential prior to PCA, as the diffusion and decay phases of the raw received signals are not derivable in Cartesian domain. As can be seen from the raw signal in Fig. 1(a), the received signals in Cartesian domain are not discriminable for different sample thicknesses, without closer inspection into the gradient of the trailing parts of the responses. To pre-process the signals, they are transformed using bi-symmetric logarithmic transformation, using

$$
V^{b i-\operatorname{symlog}}(t)=\operatorname{sgn}[V(t)] \cdot \log _{10}\left[1+\mid\left(V(t) \mid / 10^{C}\right],\right.
$$

where $\operatorname{sgn}(*)$ is the standard mathematical Sign (or Signum) function and $C$ is a scaling constant to smoothly transform the region near zero so that it remains finite. In this case, $C$ is 
chosen to be -15 , which equals to the lowest order observed in the acquired signals in the numerical models. Admittedly, analysis of the signals obtained through experiment would not be possible to be carried out in such voltage range $\left(10^{-15} \mathrm{~V}-10^{1} \mathrm{~V}\right)$. However, this bi-symmetric transformation requires the lowest order of the signal to be identified and used in (5) in order to appropriately transform the PEC signals. This transformation is also utilised instead of the conventional logarithmic transformation to avoid negative values in the signals to be treated as infinite, which conveniently allows the analysis of noise margin and probe vibration. The outcomes of these signal pre-processing steps are provided in Fig. 1(b). The signal pre-processing amplifies the differences caused by the diffusion of eddy current, represented at only the trailing part of the received signal in Cartesian domain.

\section{Modelling AND EXPERIMENTAL SETUP}

The structure of the test sample considered in this work was a carbon steel plate with a layer of insulation. 2D axisymmetric COMSOL Multiphysics models were developed, with the reference configurations: excitation coil outer diameter, $d o_{e}=80 \mathrm{~mm}$, excitation coil inner diameter, $d i_{e}=58 \mathrm{~mm}$, excitation coil height, $h_{e}=12.5 \mathrm{~mm}$, excitation coil offset, $O_{e}=2 \mathrm{~mm}$, sensing coil outer diameter, $d o_{s}=105 \mathrm{~mm}$, sensing coil inner diameter, $d i_{s}=83 \mathrm{~mm}$, sensing coil height, $h_{s}=12.5 \mathrm{~mm}$, sensing coil offset, $O_{s}=2 \mathrm{~mm}, 2$ A supply current, and number of turns of 210 for both excitation and sensing coil. The carbon steel test sample has the following parameters: relative permeability, $\mu=100$, and conductivity, $\sigma=5.5 \mathrm{MS} / \mathrm{m}$. The thickness, $d$ is varied from $4 \mathrm{~mm}$ to 12 $\mathrm{mm}$, at an increment of $2 \mathrm{~mm}$. Fig. 2 provides the visualisation of the aforementioned parameters. In this implementation, only the rising edge of the whole pulse period was considered. Transient analysis was carried out in the range of $0 \mathrm{~ms} \leq$ $t \leq 300 \mathrm{~ms}$, to allow the complete decay of the eddy current in the sample. In the case of a finite element model, since the rate of the decay is exponential, the PEC signals were considered to reach $0 \mathrm{~V}$ when it became asymptotic towards the $\mathrm{x}$-axis (at $10^{-15} \mathrm{~V}$ ).

For the purpose of experimental validation, a PEC system consisting of a PEC probe, excitation circuit and a data acquisition DAQ system was developed. The parameters of the PEC probe were the same as those in the numerical model. The supplied excitation voltage was set at $10 \mathrm{~V}$, with $8 \mathrm{~Hz}$ frequency. A total of 16 signals were acquired for each data acquisition, and averaged for white noise suppression. Similar signal transformation as (5) was later carried out, with $C=-4.5$, which equals to the smallest order of the PEC signals. The obtained PEC signals were then gated, in the range of $1.5 \mathrm{~ms} \leq t \leq 50 \mathrm{~ms}$. Arguably, this step requires two configuration parameters to be determined, which defeats the main objective of using PCA. However, the step is common in interpreting PEC signals for ferromagnetic application, where the time range can be easily determined to avoid the unnecessary peak in the early part of the signal that contains high frequency components and the later part that is highly affected by the switching on/off of the excitation

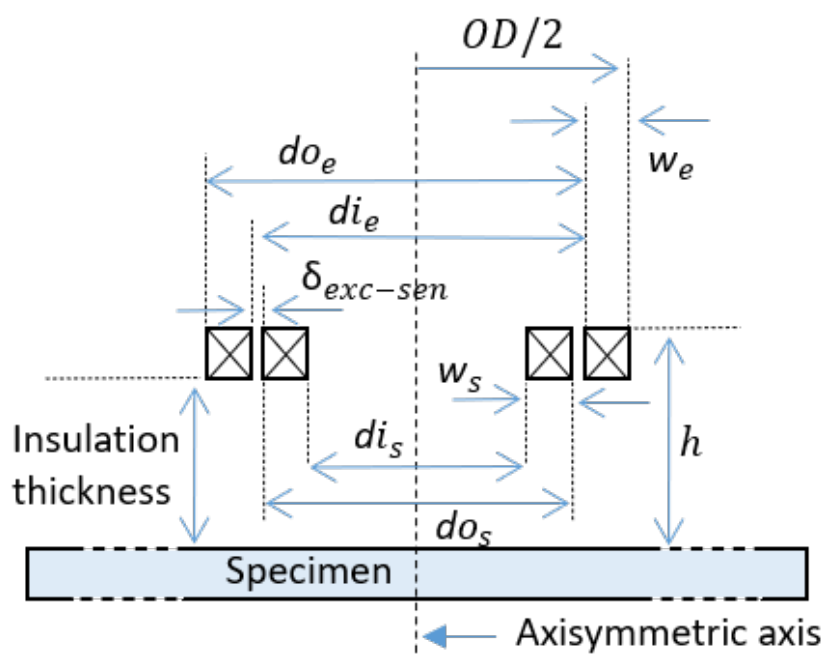

Fig. 2. Cross sectional image of the PEC setup.

current. Moreover, the PEC signal peak is typically clamped to a certain voltage level based on the DAQ device saturation, which can be used as a threshold value in data post-processing. This time-gating step, nonetheless, can still be omitted as the peak of the PEC signal can be made insignificant by the bi-symmetric logarithmic transformation step. Each test was repeated five times, where the results for these showed good repeatability in all cases.

\section{Results}

\section{A. Effects of Excitation-sensing Coil Distance}

The excitation-sensing coil distance, $\delta_{\text {exc-sen }}$ was varied over the range of $1.5 \mathrm{~mm}$ to $9.5 \mathrm{~mm}$, at intervals of $2 \mathrm{~mm}$. As explained in Section II-B, since the variation of the parameter $\delta_{\text {exc-sen }}$ affects other probe parameters, the variations of other parameters followed the premise described in the section. The responses for $4,6,8,10$, and $12 \mathrm{~mm}$ pipe thicknesses were obtained for each excitation-sensing coil distance. PCA was applied separately on the five different data sets obtained from the models. The PEC signals and scores for different pipe wall thicknesses obtained are shown in Fig. 3(a)-(b).

Selective assessments of PEC signals for $\delta_{\text {exc-sen }}$ of 1.5 $\mathrm{mm}$ and $9.5 \mathrm{~mm}$ are indicated in Fig. 3(a). Decreasing $\delta_{\text {exc-sen }}$ allows less coupling between the excitation and sensing coil, thus providing smaller amplitudes in the PEC signals. However, this probe parameter does not appear to contribute to separation of the PEC signals, and this can be evaluated from the trivial differences in the PEC signals for $\delta_{\text {exc-sen }}=1.5 \mathrm{~mm}$ as compared to $\delta_{\text {exc-sen }}=9.5 \mathrm{~mm}$.

The PCA scores for different $\delta_{e x c-s e n}$ values with varying sample thicknesses are shown in Fig. 3(b), based on which the PCA scores can be claimed to provide meaningful attribute in relation to the sample thickness. As noted, increasing sample thickness increases the PCA score. However, varying this parameter does not affect the sensitivity of the probe across all thicknesses, where the slope of the PCA score for each $\delta_{\text {exc-sen }}$ does not show any significant difference between 


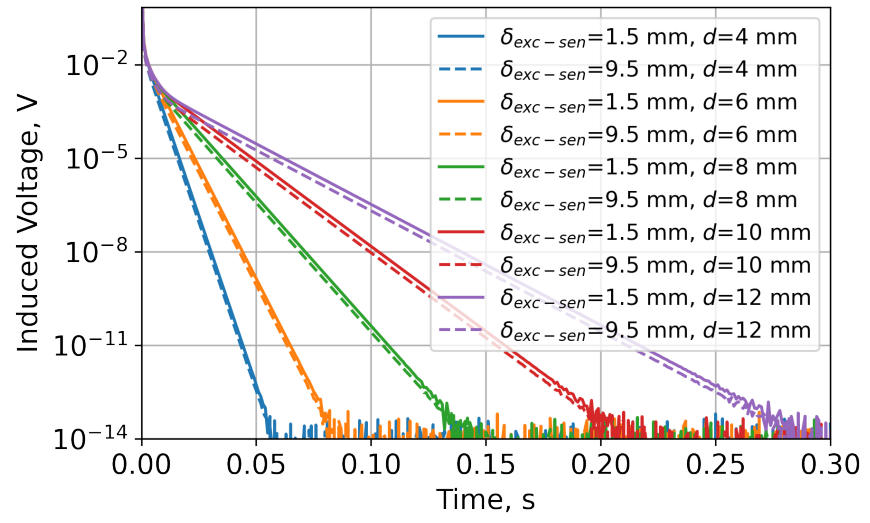

(a)

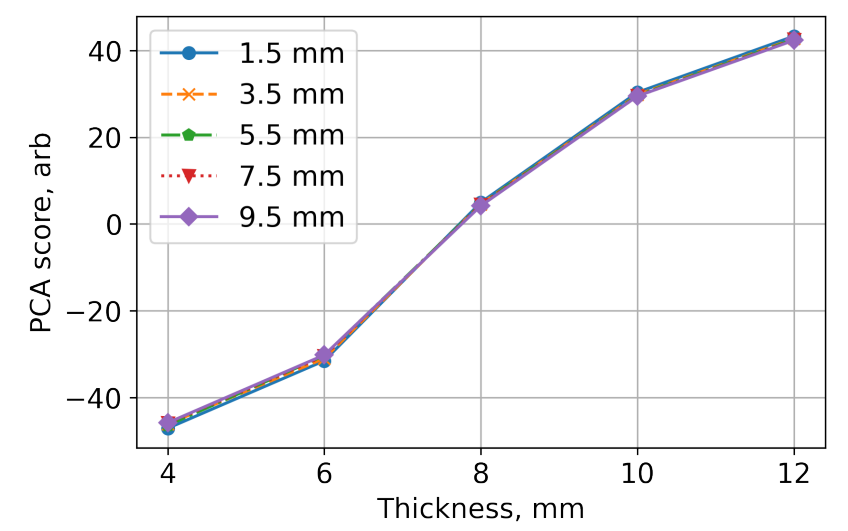

(b)

Fig. 3. (a) PEC responses of different sample thicknesses, $d$, for different $\boldsymbol{\delta}_{\text {exc-sen }}$ values (after bi-symmetric logarithmic transformation), and (b) their corresponding PCA scores.

each other. This essentially provides information regarding the minimal contributions of $\delta_{\text {exc-sen }}$ towards the sensitivity of the PEC system.

A further notable observation is the values of the PCA scores, which are approximately consistent throughout the different $\delta_{\text {exc-sen }}$ values, even though the signals' amplitudes are different for different values of $\delta_{\text {exc-sen }}$. As previously mentioned, the assessment, however, represents the relative differences between the PCA scores for each $\delta_{e x c-s e n}$, without information on the absolute values of the PEC signals' amplitudes. Further evaluation of the amplitudes of PEC signals corresponding to different $\delta_{\text {exc-sen }}$ values can be carried out by examining the PEC signals in Fig. 3(a).

\section{B. Effects of Sensing Coil Outer Diameter}

To establish the effects of the sensing coil outer diameter, this parameter was changed from $75 \mathrm{~mm}$ to $105 \mathrm{~mm}$, at intervals of $10 \mathrm{~mm}$. While doing so, $\delta_{\text {exc-sen }}$ was kept constant at $1.5 \mathrm{~mm}$. Similar to the methodology used in the previous section, in varying $O D$, the same premise explained in Section II-B was applied. The corresponding results from the numerical modelling are presented in Fig. 4.

The responses of two different sensing coils, $O D$, corresponding to different sample thicknesses are shown in

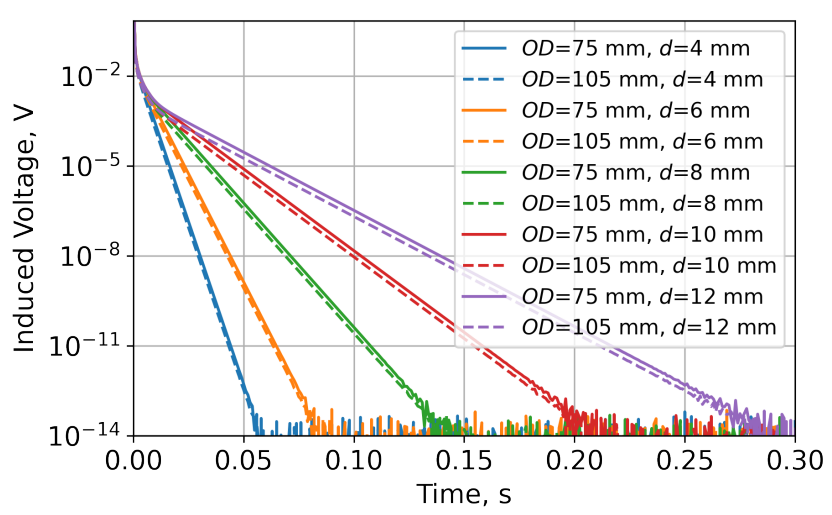

(a)

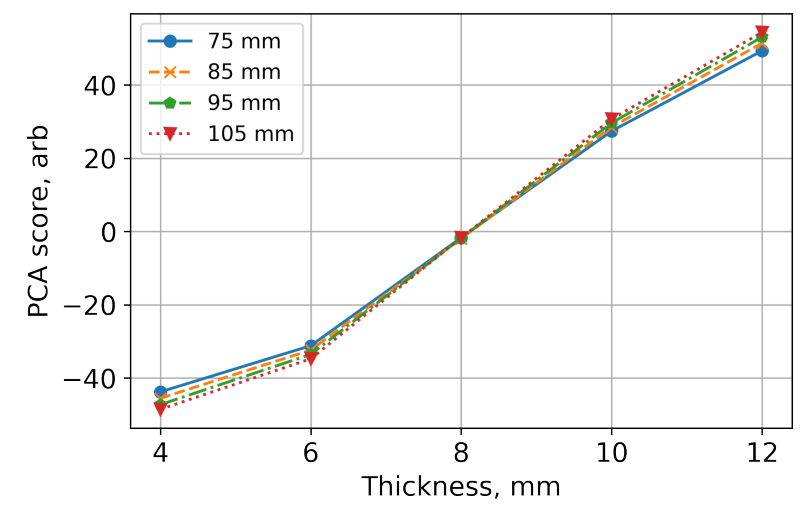

(b)

Fig. 4. (a) PEC responses of different sample thicknesses, $\boldsymbol{d}$, for different $\boldsymbol{O D}$ values (after bi-symmetric logarithmic transformation), and (b) their corresponding PCA scores.

Fig. 4(a). The signal amplitude evidently increased with respect to $O D$. Although it should be noted that the numerical models did not consider the effect of the DC resistance of the sensing coil, this relationship is expected to be consistent in the experiment. Bigger coil allows bigger volume to be subjected to changes in magnetic field, which allows more electromotive force (emf) generation. The responses for outer diameter of $105 \mathrm{~mm}$ probe also appeared to exhibit higher deviations across the sample thicknesses, as compared to their counterparts for $75 \mathrm{~mm}$. It should further be noted that the simulation did not consider 'lossy' characteristics of the inspected sample and the limit of depth of penetration, where these two attributes might contribute to different results in the experiments.

Quantitative analysis can be seen in the results of the PCA scores in Fig. 4(b). Unlike $\delta_{e x c-s e n}$, the results clearly show the higher variances obtained for PEC signals of bigger coils. This is because, bigger coil supplies magnetic field that is more diverged as compared to the supplied magnetic field by smaller coil. Further observation into this phenomenon is provided in Fig. 5, where the distribution of induced eddy current within the $12 \mathrm{~mm}$ sample is visualised for OD of $75 \mathrm{~mm}$ and $105 \mathrm{~mm}$ at $2 \mathrm{~ms}$ (before eddy current diffuses to the inner specimen's surface). It is clearly shown that the distribution of the induced eddy current in the sample for coil of $75 \mathrm{~mm}$ OD is more 
converged than that of coil of $105 \mathrm{~mm}$ OD. This essentially proves the higher sensitivity of bigger coils towards changes in the sample parameters. Hence, it is more favourable to design the coil to have as large diameter as possible, while at the same time, careful consideration is given to not allow the footprint size to be considerably larger than the expected size of defect.

\section{Experimental Validations}

The results show that the $\delta_{e x c-s e n}$ contributes to relatively negligible variations to the separation of the PEC signals. Comparatively, $O D$ parameter shows significance for the sensitivity of the PEC system, so this parameter was varied in the experimental analysis. The acquired signals are shown in Fig. 6. The differences in the PEC signals amplitudes for different $O D$ are expected, as inferred in Section IV-B. As noted, the amplitudes of the responses from experiment did not match the ones from the numerical modelling, attributed by the difference in the inductance value and the wire resistance that are not considered in the numerical modelling. Nevertheless, the trends of the signals show good agreement between the experimental and numerical model results.

What stands out in the experimental results is the smaller variances in the signals acquired by $75 \mathrm{~mm}$ probe, as compared to $105 \mathrm{~mm}$ probe. The signals for thicker samples $(10 \mathrm{~mm}$ and $12 \mathrm{~mm}$ ) for $75 \mathrm{~mm}$ probe are also not easy to be differentiated, making it difficult for thickness interpretation. This is because, comparatively, the supplied magnetic field for smaller probes is more convergent, allowing the field to only travel to a shallow depth within the samples. This effect due to penetration depth limitation is not obvious in the modelling results, as the mathematical models try to approach the specified convergence tolerance, making the signals to be discriminable in the logarithmic scale. At the same time, limitations in the experimental setup (e.g. lossy medium, discretisation limits in analog-to-digital convertor, high white noise margins) are inevitable and provide more constraint to the data acquisition accuracy.

Following the approaches taken in obtaining the simulation results, the scores obtained are presented in Fig. 7. The scores, against the sample thickness, confirms the recognised trends

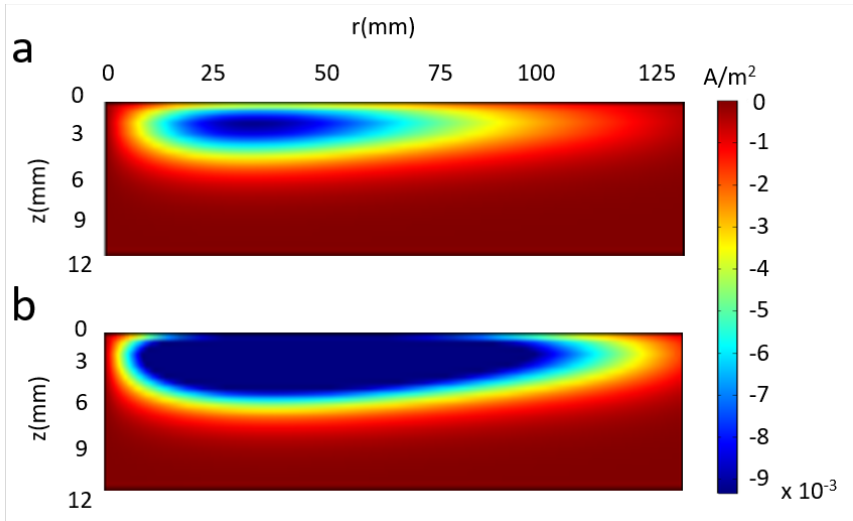

Fig. 5. The distributions of induced eddy current in azimuthal direction for (a) coil of $75 \mathrm{~mm} O D$ and (b) coil of $105 \mathrm{~mm} O D$ at $2 \mathrm{~ms}$.

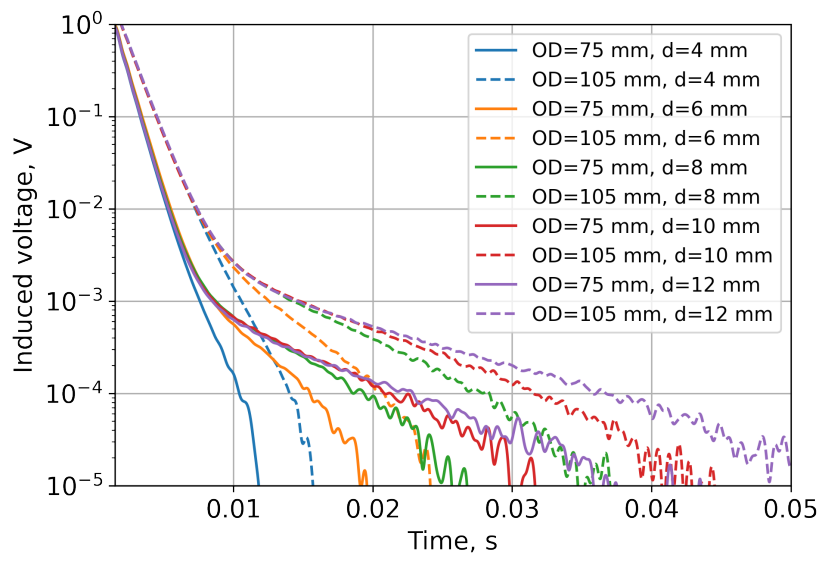

Fig. 6. PEC responses of different sample thicknesses, $\boldsymbol{d}$, for $\boldsymbol{O D}$ of $75 \mathrm{~mm}$ and $105 \mathrm{~mm}$ from experiments (after bi-symmetric logarithmic transformation).

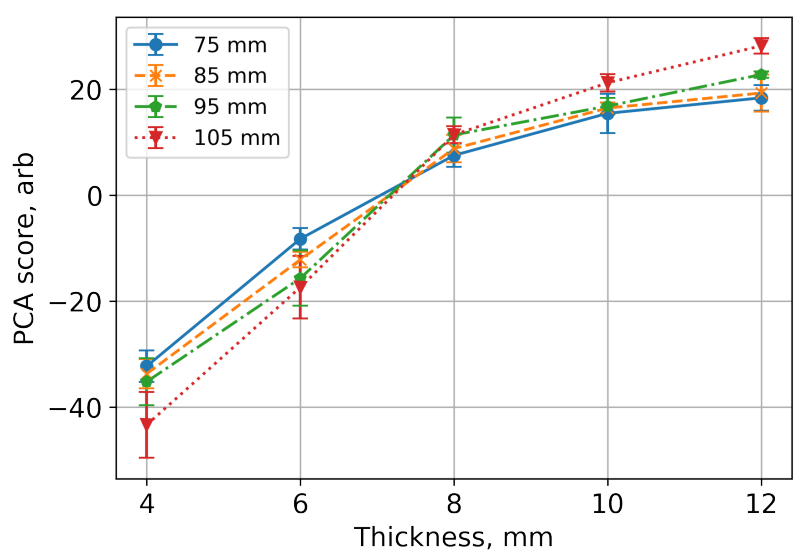

Fig. 7. PCA scores for different $\boldsymbol{O D}$ corresponding with different $\boldsymbol{d}$ from experiments.

in the simulation study. Discrepancies in the graph can be observed, due to the aforementioned depth of penetration limitations and noises. Compared to the simulation results, experimental results also exhibit higher separation between each score, which was potentially contributed by the differences in the inductance values of both coils. Another significant difference observed is by comparing the trends in experimental results with those of simulation results. In general, both experimental and simulation results exhibit an increasing trend of scores with respect to thickness, but the slopes are different. Simulation results, despite the probe diameters, have more linear slopes when compared to the experimental results. For experimental results, the scores for smaller probes (especially $75 \mathrm{~mm}$ ) depreciate in their capabilities to differentiate thick samples. The slope across $8 \mathrm{~mm}$ to $12 \mathrm{~mm}$ for $75 \mathrm{~mm}$ probe gradually declines, representing the limit of the depth of penetration. It is thus evident to suggest that the $75 \mathrm{~mm}$ probe is limited to thin samples. Nevertheless, this parametric study still suggests higher variances for larger probe diameter, which again adheres to the results simulated in Section IV-B. 


\section{CONCLUSION}

A new feature extraction method using PCA has been investigated. The PCA scores obtained from the simulation results show that the distance between the excitation-sensing coil is not imperative in determining the sensitivity of the probe. The excitation coil diameter, however, contributes largely to the sensitivity of the PEC probe. Moreover, the results have demonstrated the quantitative capability of the PCA technique. Experimental validations have further substantiated the use of PCA in the design stage of a PEC probe. The application of this proposed technique is certainly not limited to the two probe parameters studies, but can be extended to evaluate further parameters such as the width and height of coils as well as excitation current parameters.

\section{ACKNOWLEDGMENT}

The authors would like to thank Lloyd's Register Foundation for funding of this research. Lloyd's Register Foundation helps to protect life and property by supporting engineering related education, public engagement and the application of research.

\section{REFERENCES}

[1] K. N. Azaman, A. Sophian, and F. Nafiah, "Effects of coil diameter in thickness measurement using pulsed eddy current non-destructive testing," IOP Conference Series: Materials Science and Engineering, vol. 260, no. 1, pp. 1-6, Aug. 2017.

[2] S. Majidnia, R. Nilavalan, and J. Rudlin, "A numerical study of the depth of penetration of eddy currents," Insight-Non-Destructive Testing and Condition Monitoring, vol. 58, no. 3, pp. 129-134, Mar. 2017.

[3] D. Zhou, G. Y. Tian, and Y. Li, "Simulation based on optimisation of pulsed eddy current probe design," Nondestructive Testing and Evaluation, vol. 25, no. 3, pp. 219-230, Jan. 2010.

[4] Z. Xu, X. Wu, C. Huang and H. Ke, "Analysis of excitation current parameters in pulsed eddy current testing for ferromagnetic metallic materials," International Journal of Applied Electromagnetics and Mechanics, vol. 43, no. 7, pp. 648--653, Oct. 2012.

[5] C. Huang, X. Wu, Z. Xu, and Y. Kang, "Ferromagnetic material pulsed eddy current testing signal modeling by equivalent multiple-coilcoupling approach," NDT \& E International, vol. 44, no. 2, pp. 163168, Mar. 2011

[6] Y. He, G. Y. Tian, H. Zhang, M. Alamin, A. Simm, and P. Jackson, "Steel Corrosion Characterization Using Pulsed Eddy Current Systems," IEEE Sensors Journal, vol. 12, no. 6, pp. 2113-2120, Aug. 2012.

[7] C. P. Dolabdjian, L. Perez, V. O. De Haan, and P. A. De Jong, "Performance of Magnetic Pulsed-Eddy-Current System Using High Dynamic and High Linearity Improved Giant MagnetoResistance Magnetometer," IEEE Sensors Journal, vol. 6, no. 6, pp. 1511-1517, Dec. 2006.

[8] Y. Kitane, S. Ando, Y. Itoh, and Y. Nakano, "Numerical Study On Pulsed Eddy Current Thickness Measurement For Steel Plates With Thickness Loss," in Proc. EASEC-14, Ho Chi Minh, Vietnam, 2016.

[9] S. M. van den Berg, "Modelling and Inversion of Pulsed Eddy Current Data," Ph.D. dissertation, Department of Electrical Engineering, Mathematics and Computer Science, Delft University of Technology, Delft, Netherlands, 2003.

[10] N. Ulapane, A. Alempijevic,T. V. Calleja and J. V. Miro, "Review of Pulsed-Eddy-Current Signal Feature-Extraction Methods for Conductive Ferromagnetic Material-Thickness Quantification," Electronics, vol. 8, no. 5, pp. 470, Apr. 2019.

[11] K. F. Faurschou, P. R. Underhill, J. Morelli, and T. W. Krause, "Pulsed eddy current probe optimization for steel pipe wall thickness measurement," AIP Conference Proceedings, vol. 2102, no. 1, pp. 080002, May. 2019.

[12] T. Ding, Z. Yang, H. Huang, P. Huang, D. Hou, and G. Zhang, "A method for characterizing defects in multi-layer conductive structures by combining pulsed eddy current signals with PCA components," AIP Conference Proceedings, vol. 2102, no. 1, pp. 080003, May. 2019.
[13] J. A. Buck, P. R. Underhill, S. G. Mokros, J. E. Morelli, V. K. Babbar, B. Lepine, J. Renaud, and T. W. Krause, "Pulsed Eddy Current Inspection of Support Structures in Steam Generators," IEEE Sensors Journal, vol. 15, no. 8, pp. 4305-4312, Aug. 2015.

[14] P. F. Horan, P. R. Underhill, T. W. Krause, "Real Time Pulsed Eddy Current Detection of Cracks in F/A-18 Inner Wing Spar Using Discriminant Separation of Modified Principal Components Analysis Scores," IEEE Sensors Journal, vol. 14, no. 1, pp. 171-177, Jan. 2014.

[15] C. A. Stott, P. R. Underhill, V. K. Babbar, and T. W. Krause, "Pulsed Eddy Current Detection of Cracks in Multilayer Aluminum Lap Joints," IEEE Sensors Journal, vol. 14, no. 1, pp. 171-177, Jan. 2014.

[16] V. O. D. Haan, P. A. D. Jong, L. Perez, and C. Dolabdjian,, "Towards Material Characterization and Thickness Measurements using Pulsed Eddy Currents Implemented with an Improved Giant Magneto Resistance Magnetometer," in Proc. ECNDT-2006, Berlin, Germany, 2006.

[17] A. Sophian, G. Y. Tian, D. Taylor, J. Rudlin, "A feature extraction technique based on principal component analysis for pulsed Eddy current NDT," NDT \& e International, vol. 36, no. 1, pp. 37-41, Jan. 2003.

[18] M. Alamin, G. Y. Tian, A. Andrews P. Jackson, "Principal component analysis of pulsed eddy current response from corrosion in mild steel," IEEE Sensors Journal, vol. 12, no. 8, pp. 2548-2553, Aug. 2012.

[19] I. T. Jolliffe, and J. Cadima, "Principal component analysis: a review and recent developments," Philosophical Transactions of the Royal Society A: Mathematical, Physical and Engineering Sciences, vol. 374, no. 2065, pp. 20150202, Apr. 2016. 